\title{
Advanced Signal Processing Approaches for Quadratic Frequency Modulated Thermal Wave Imaging
}

\author{
B. Suresh, M. Manorama, M. M. Bhupesh, K. Sai Kiran, G. V. P. Chandra Sekhar Yadav, V. S. Ghali \\ Infrared Imaging Center, Department of Electronics and Communication Engineering, Koneru Lakshmaiah \\ Education Foundation, Vaddeswaram, AP, India.
}

\begin{abstract}
Non-stationary thermal wave imaging (NSTWI) which is one of the techniques of Infrared Thermal Non-Destructive Testing (IRTNDT) plays a vital role in inspecting, evaluating and analyzing various materials. With excellent capabilities for surface and sub-surface crack and back holes detection, moderate peak power and taking less time in analyzing results when compared to lock in thermography and pulsed thermography, NSTWI excel in this domain. This paper highlights comparison of various NSTWI post-processing techniques, further experimentation is done to detect defects in composites. Signal to noise ratio of each post processing technique is considered and the best technique is chosen.
\end{abstract}

Key words : Infrared Thermal Non-Destructive Testing, Signal to noise ratio, Non-stationary thermal wave imaging.

\section{INTRODUCTION}

Detection of back holes and cracks in metals are particularly important in the quality analysis of metal specimen as they can be a starting point for severe damage. The performance of the components used is extremely important in many industrial divisions, especially in the manufacture of safety critical components [23]. Non-destructive testing is the analysis of discontinuity materials, without destroying components of the sample. The element can still be used after careful examination of the sample [16]. Present non-destructive tests are widely used to ensure distribution, production performance, monitor manufacturing processes reduce production costs and keeps a consistent level of quality during the manufacturing processes.

Thermal radiation-based infrared imaging has emerged in recent decades as an efficient, fast and wide inspection area. Non-destructive infrared testing (IRNDT) $[13,18]$ is considered to be a versatile testing process with the potential for testing detecting defects in materials such as composites, metals and semiconductors. Compared to other NDT techniques, the main advantage of thermographic inspection is that it can observe a large area in a short period [22].

Both qualitative and quantitative sub-surface tests, it uses temporal temperature evolution over the sample body. It can be split into active and passive strategies based on the process of bringing in confusion before scanning. For passive thermography analysis, the natural surface temperature evolution of the sample body is specifically considered compared to its equivalents for identification of defects for evaluation and irregular area thermal signature $[1,5]$. Temporal signature is used for quantitative analysis. Active thermography therefore uses the evolution of surface temperature after stimulation of the external source [26, 27]. Infrared non-destructive testing (IRNDT) is gaining attention because of its non-contact, non-invasive and wide area inspection capabilities. Among the existing IRNDT techniques, pulse thermography is noted for simplicity and well-established methodology. Pulsed phase thermography uses Fourier transforms in thermal response collected after pulsed simulation to represent subsurface irregularities to unscramble waves of different frequencies. FMTWI can solve issues associated with methods such as peak energy in repeated LT testing.

In lock in thermography, the specimen is excited by low power and low frequency sinusoidal periodic excitation as low frequencies are less attenuated compared to high frequencies and travels deeper into the test object. LT uses harmonic heat to energize the sample surface at low-power, enabling us to calculate amplitude and phase angle.

For Quadratic frequency modulated wave imagery (QFMTWI), more energy is concentrated at low frequencies rather than high frequencies, unlike linearly modulated wave imaging, which enables better depth detection than its linearly modulated counterpart [3]. It uses a quadratic sweep of a frequency band applied to solve the anomalies at different depths in a single experimental period for a finite duration at low peak powers. Therefore, quadratic modulated excitation provides more heat deposition, better depth resolution

\section{THEORY}

\subsection{Quadratic FMTWI}

In Quadratic frequency modulated thermal wave imaging, the surface of the test specimen is provided with a QFM chirped stimulation that consequently generates thermal waves which are similar and closer to the surface that perpetuate into the object's interior $[4,25]$. Such thermal waves that migrate, 
further reflective from boundary and lead to an increase in temperature across the surface of the body. The temperature reaction which is accomplished by $1 \mathrm{D}$ thermal equation is:

$$
\frac{\partial^{2} T}{\partial x^{2}}=\frac{1}{\alpha} \frac{\partial T}{\partial t}
$$

Where $\alpha$ is diffusion coefficient; $\mathrm{T}$ is the instant temperature at depth $\mathrm{x}$ and time $\mathrm{t} ; \mathrm{L}$ is the thickness of sample ;

Quadratic frequency-modulated heat flux, using the boundary conditions given by:

$$
\begin{aligned}
& \left.\frac{\partial T}{\partial t}\right|_{x=0}=Q_{0} e^{-j 2 \pi\left(a t+b t^{3}\right)} \\
& \left.\frac{\partial T}{\partial t}\right|_{x=L}=0
\end{aligned}
$$

Where Q0 is the incident heat flux magnitude; $\mathrm{k}$ is the material's thermal conductivity; initial frequency is $\mathrm{f} 0$; $b$ is the sweeping speed.

Temperature at depth $\mathrm{x}$ is obtained when solving the heat equation using the boundary conditions as described in Equation $(2,3)$ as:

$$
\begin{aligned}
& T(x, \mathrm{t})=Q_{0} e^{-x / \mu}[\cos (k)+j \sin (k)] \\
& k=2 \pi\left(a t+\frac{b t^{2}}{2 T}\right)-x / \mu
\end{aligned}
$$

These propagating waves, disturbed by a sub-surface discontinuity, contribute to a localized temperature variation upon the test specimen's surface at the relevant locations and further used for detection.

The length of thermal diffusion for QFMTWI is as follows:

$$
\mu=\sqrt{\frac{\alpha}{\pi(a+b t / T)}}
$$

From Equation (5), it is evident that the $\mu$ dependency on $t$ indicates that QFMTWI is capable of analysing defects at different depths within a single test period and thus providing sub-surface depth scanning.

\subsection{Pre Processing}

The thermal responses of thermal profiles are generated by applying either linear fitting or discrete Fourier transform (DCT). The linear fitting technique eliminates thermal response's stationary portion as it increases linearly over time. Over these mean thermal profiles different processing methods will be applied and the corresponding sub-surface details will be extracted.

\subsection{Post Processing Techniques}

Through these pre-processed thermal profiles, different processing methods such as frequency domain phase analysis, time domain phase, pulse compression, principal component comparison etc. are used to detect underground phenomena with improved contrast [15].

\section{A. Phase Analysis}

A Fourier transform is applied in phase analysis to each pixel's dynamic response and the resulting phase data is obtained using (6) and If $\operatorname{Re}(\mathrm{F}(\mathrm{n}))$ is the real part of the nth element and $\operatorname{Im}(\mathrm{F}(\mathrm{n}))$ is the imaginary part of the nth element of the $f[n]$ transformation [9] of Fourier then duration is calculated at any frequency from (7)

$$
\begin{gathered}
F(\omega)=\sum_{n=0}^{N-1} f(n) e^{j \omega n}=\sum_{n=0}^{N-1} \operatorname{Re}(F(n \omega))+\operatorname{Im}(F(n \omega)) \\
\phi(n)=\tan ^{-1}\left(\frac{\operatorname{Im}(F(n \omega))}{\operatorname{Re}(F(n \omega))}\right)
\end{gathered}
$$

\section{B. Correlation}

Pulse compression is a tool of evaluating the time domain. Details of a pixel in captured thermograms are ordered in this method in a series called the pixel's temporal thermal profile. With all the pixels in view, a similar procedure is replicated and corresponding thermal profiles are created for all the pixels [24].

Both profiles include the temporal thermal response relating to the excitation offset and the adaptive response according to the excitation being proposed. To obtain only the corresponding dynamic response, the offset is removed from each profile using an appropriate data fitting procedure (a linear fitting procedure is generally to be adopted). By taking the average of a few randomly selected mean excluded non-defective pixel profiles, a reference profile is created [8].Between the mean thermal profiles of each and every pixel and the reference pixel, cross-correlation is done in the next stage. This cross-relation results in a standardized data sequence of correlation. For all the pixel profiles in view, a similar correlation profile is calculated [21].

These profiles have been rearranged so that at the delayed moment the correlation coefficients of every pixel which are normalized are stored in their respective spatial positions [17, 20] to form correlation picture. As shown, due to their reliance on delay and attenuation corresponding to the defect profiles, they can provide a spatial variance at defect locations. This contrast coefficient was used to detect defects in comparison images. This technique for identifying defects is similar to the time domain thermography study of Pulse thermography [14]. But the additional benefits gained with this approach are noise removal while processing and improving SNR [19].

The cross correlation of the thermal response is given by $\mathrm{R}(\mathrm{t})$.

$$
R(t)=\int_{-\infty}^{\infty} h(t) g(t+\tau) d t
$$

Where $g(t)$ and $h(t)$ are thermal response and preselected thermal response respectively. 
B. Suresh et al., International Journal of Emerging Trends in Engineering Research, 7(11), November 2019, 599- 603

\section{Principal component analysis}

Principal component analysis is method used to project data orthogonally and to reduce dimensional linear space which is also called as sub-space hence increasing the projected data's variance. It optimizes the variance.

Main components are determined by performing multiplication between each column of own vector matrix and original data matrix [23]. Eventually, the main element matrix reconstructs a 3D sequence.

The projection of these Eigen vectors $\mathrm{V}$ into the original data $\mathrm{S}$ is given by

$$
P C s=\sum_{n=1}^{L} V^{T} S \text { where } L<<N
$$

\section{Orthonormal projection Transform}

Study of orthonormal projection uses the algorithm of Gram-Schmidt to produce orthonormal random vectors, and thermal profiles are also mapped onto them to obtain normalized random coefficients of projection.

3D thermal history is initially transformed into a two-dimensional matrix in this approach, the vectors of which are produced by reorganizing different thermogram pixel values. This is replicated with all the thermograms collected in various instances, i.e. a thermogram series recorded at the rate of 25 frames per s for $100 \mathrm{~s}$ provides 2500 thermograms each with a resolution of 320 / 250 resulting in a matrix row vector of 80,000 elements with 2500 elements in each column [7].

Between them, a first column vector $\mathrm{f}_{1}(\mathrm{n})$ is normalized and a first random vector is generated using V1 $=f_{1}[n] /\left\|f_{1}[n]\right\|$ somewhere between the two columns [6]. This standardized random vector is then used with the next column vector $f_{2}(n)$ to produce the next random vector $\mathrm{V} 2$, as shown in the equation below:

$$
\mathrm{v}_{2}=\mathrm{f}_{2}[\mathrm{n}]-\left(\mathrm{v}_{1} \mathrm{~T}_{2}[\mathrm{n}]\right) \mathrm{v}_{1}
$$

The process continues with all column vectors and the resulting random vectors are produced to formulate a random matrix produced from the Gram-Schmidt algorithm with orthonormal base vectors. The random matrix columns are constructed with two-dimensional thermal data. In addition, the matrix of projection coefficients corresponding to each random vector was rearranged to form random projection images with a resolution [10] equal to a thermogram to define subsurface anomaly indications using the contrast coefficient of projection.

\section{EXPERIMENTATION}

A $16 \mathrm{~cm} X 16 \mathrm{~cm} X 0.5 \mathrm{~cm}$ GFRP specimen with flat back holes of various diameters at different depths is designed and simulated with quadratic frequency modulated thermal flux at front plane in COMSOL Multiphysics software.

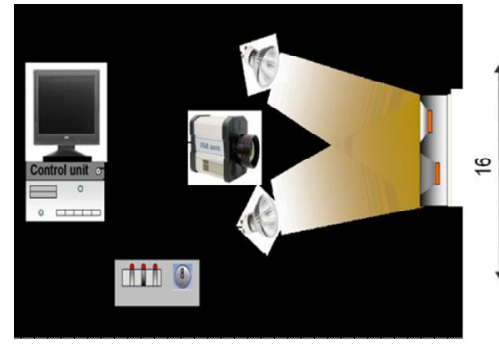

a

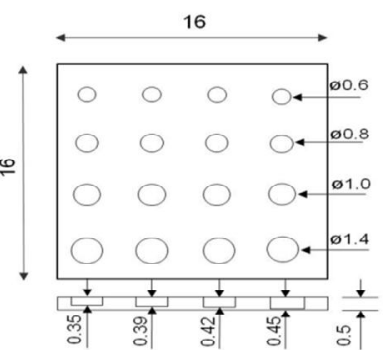

$\mathrm{b}$
Figure 1: a. Experimental schematic of QFMTWI and b. Layout of GFRP specimen (all dimensions in $\mathrm{mm}$ ). Specimen layout is shown in fig 1 . b. A $2 \mathrm{KW}$ excitation of quadratic frequency modulated with a range of frequencies starting from a range of frequencies 0.01-0.1 hertz is imposed for a time of 100 seconds. Then, thermal response from the surface of the material is captured at the rate of 25 frames per sec. This captured thermal response has been processed with various post processing techniques employed to extract the subsurface details.

\section{RESULTS AND DISCUSSION}

On the acquired thermal response, FFT phase, Pulse compression, principal component analysis and orthonormal projection analysis techniques are employed. Results shown in fig. 2.a is FFT phase at $0.027 \mathrm{~Hz}$, fig. 2.b is Pulse compression at $16.08 \mathrm{sec}$, fig. 2.c is $2 \mathrm{nd} \mathrm{PCA}$ and fig. 2 .d is $1 \mathrm{st}$ random projection component. From the results, it is observed that random projection transform provides better results in defect visualization compared to other processing schemes.

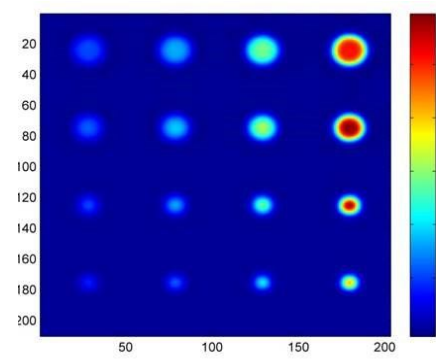

a

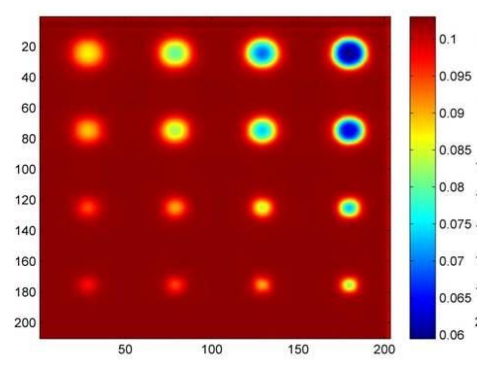

C

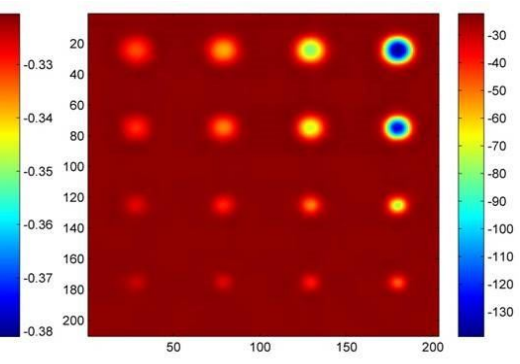

b

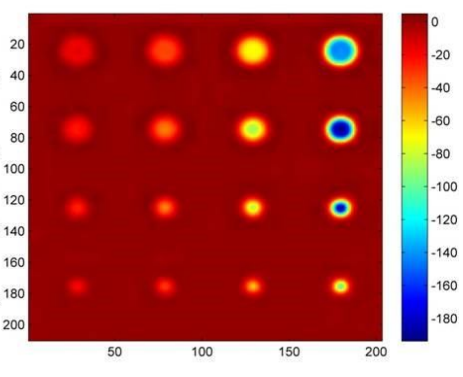

d
Figure 2: a. FFT phase at $0.027 \mathrm{~Hz}$, b. Pulse compression at $16.08 \mathrm{sec}, \mathrm{c} .2 \mathrm{nd}$ principal component and $1 \mathrm{st}$ random projection component for raw thermal response. 
B. Suresh et al., International Journal of Emerging Trends in Engineering Research, 7(11), November 2019, 599- 603

Further signal to noise ratio is employed on each defects for afore mentioned processing techniques. SNR is the ratio between differences in means of defective region to non-defection region divided by the value non-defective region's standard deviation. The Signal to noise values are represented in below fig. 3 , inferring that random projection transform gives better signal to noise ratios.

$$
S N R(d B)=20 \log \left(\frac{\mu_{\text {Defective }}-\mu_{\text {Non-Defective }}}{\sigma_{\text {Non-defective }}}\right)
$$

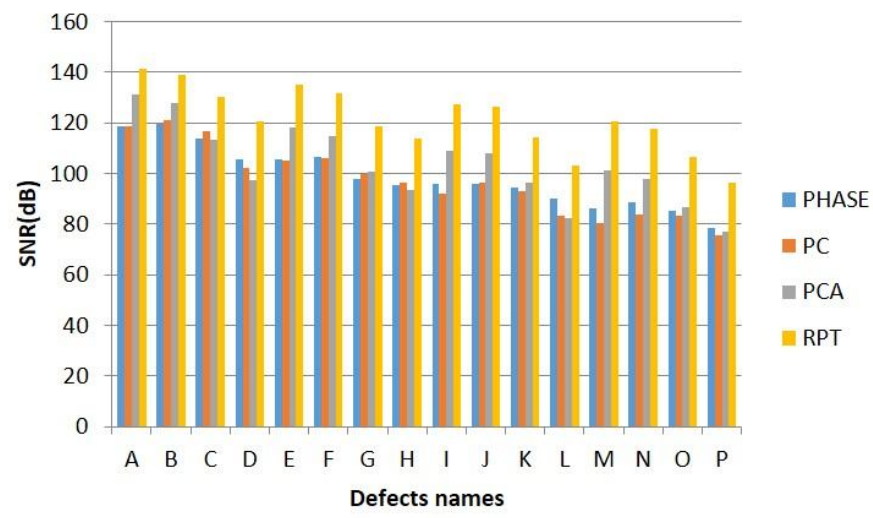

Figure 3: Signal to noise ratios of different post processing techniques employed on GFRP sample

\section{CONCLUSION}

In this paper, pre-processing on a GFRP sample which is having flat back holes is initially implemented and extracted the thermal response of it through applying Quadratic frequency modulated thermal flux. Then on that thermal response, various post processing techniques were applied. By observing the results, it is concluded that Orthonormal projection analysis gives better results as it has good defect detection capabilities and improved signal to noise rations than other post processing techniques.

\section{REFERENCES}

1. Subhani S.K., Suresh B, Ghali V.S., Empirical mode decomposition approach for defect detection in non-stationary thermal wave imaging, $N D T$ and $E$ International, Vol. 81, 2016

https://doi.org/10.1016/j.ndteint.2016.03.004

2. G.V. Subbarao and J.L. Ravipati, An improved image denoising method using compressive sensing based thresholding method, JARDCS, Vol. 10, 2018.

3. Maldague X P V, Theory and Practice of Infrared Technology for Non-Destructive Testing, New York: Wiley, 2001.

4. Suresh B., Subhani Sk, Ghali V.S., Mulaveesala R., Subsurface detail fusion for anomaly detection in non-stationary thermal wave imaging, Insight: Non-Destructive Testing and Condition Monitoring, Vol. 59, Issue. 10, 2017. https://doi.org/10.1784/insi.2017.59.10.553

5. Suresh B., Subhani S., Vijayalakshmi A., Vardhan V.H., Ghali V.S., Chirp $Z$ transform based enhanced frequency resolution for depth resolvable non stationary thermal wave imaging, Review of Scientific Instruments, Vol. 88, Issue. 1, 2017 https://doi.org/10.1063/1.4973192

6. Vanita Arora, Ravibabu Mulaveesala, Anju Rani \& Anshul Sharma, Digitised Frequency Modulated Thermal Wave Imaging for Non-destructive Testing and Evaluation of Glass Fibre Reinforced Polymers, Nondestructive Testing And Evaluation, 2018.

7. Javed Ahmad, Aparna Akula, Ravibabu Mulaveesala, and H. K. Sardana, Barker-Coded Thermal Wave Imaging for Non-Destructive Testing and Evaluation of Steel Material, IEEE Sensors Journal, Vol. 19, Issue. 2, 2019.

https://doi.org/10.1109/JSEN.2018.2877726

8. PPT Ibarra-Castanedo C. and Maldague X. "Pulsed Phase Thermography Reviewed, Proceedings of QIRT 7 -Quantitative Infrared Thermography, 2004.

9. S. Pickering, D. Almond, Matched excitation energy comparison of the pulse and lock-in thermography NDE techniques, NDT E Internat., Vol. 41, Issue. 7, 2008.

10. J.L Ravipati, A Vijayalakshmi,B.Chandana and V.S.Ghali, Multi Resolution based Enhanced Subsurface Analysis in thermal Wave Detection and Ranging, JARDCS, Vol. 9, 2017.

11. V Arora, J A Siddiqui, R Mulaveesala and A Muniyappa, Hilbert transform-based pulse compression approach to infrared thermal wave imaging for sub-surface defect detection in steel material, Insight, Vol. 56, Issue. 10, 2014 https://doi.org/10.1784/insi.2014.56.10.550

12. V.S.Ghali, P.S.S.Balaji and R. Mulaveesala, Barker coded thermal wave imaging for defect detection in carbon fibre-reinforced plastics, Insight, Vol. 53, 2011.

13. R Mulaveesala, V S Ghali and V Arora, Applications of non-stationary thermal wave imaging methods for characterization of fiber-reinforced plastic materials, Electronic letters, Vol. 49, Issue. 2, 2013. https://doi.org/10.1049/el.2012.3844

14. Sivavaraprasad Gampala, D. Venkata Ratnam and Yuichi Otsuka, Multicomponent Analysis of Ionospheric Scintillation Effects Using the Synchrosqueezing Technique for Monitoring and Mitigating their Impact on GNSS Signals, Journal of Navigation, Vol. 72, Issue. 3, 2018.

15. Ravibabu Mulaveesala, Jyani Somayajulu Vaddi, and Pushpraj Singh, Pulse compression approach to infrared nondestructive characterization, Review of Scientific Instruments, Vol. 79, 2008. https://doi.org/10.1063/1.2976673

16. V.S.Ghali, S.Sukumar, D.L.Srinivas, K.L.Mallika and Sk.Subani, Principal component non stationary 
B. Suresh et al., International Journal of Emerging Trends in Engineering Research, 7(11), November 2019, $599-603$

thermal wave imaging, International journal of systems and Technologies, Vol. 6, Issue. 2, 2013.

17. Vladimir P.Vavilov and Douglas D.Burleigh, Review of pulsed thermal NDT: Physical principles, theory and data processing, NDT\&E International, Vol. 73, 2015.

18. S.Subhani, B.Suresh and V.S.Ghali, Orthonormal Projection approach for depth - resolvable subsurface analysis in non - stationary thermal wave imaging, Insight, Vol. 58(1), 2016.

19. Deboshree Roy, Prabhu Babu, and Suneet Tuli, Sparse Reconstruction-Based Thermal Imaging for Defect Detection, IEEE Transactions On Instrumentation And Measurement, Vol. 68 , Issue. 11, 2019

https://doi.org/10.1109/TIM.2018.2889364

20. Ravibabu Mulaveesala and Suneet Tuli, Theory of frequency modulated thermal wave imaging for non-destructive subsurface defect detection, Applied Physics Letters, Vol. 89, 191913, 2006.

21. Vijaya Lakshmi A., Gopitilak V., Muzammil Parvez M., Subhani S.K., Ghali V.S., Artificial neural networks based quantitative evaluation of subsurface anomalies in quadratic frequency modulated thermal wave imaging, Infrared Physics and Technology, Vol. 97, 108-115, 2019.

https://doi.org/10.1016/j.infrared.2018.12.013

22. Kishore P.V.V., Kumar D.A., Goutham E.N.D., Manikanta M. , Continuous sign language recognition from tracking and shape features using Fuzzy Inference Engine, Proceedings of the 2016 IEEE International Conference on Wireless Communications, Signal Processing and Networking, Wisp NET, 2165 2170, 2016.

23. Anil Kumar D., Kishore P.V.V., Venkatram N., Leela Rani B., Hasitha A., Sabarish T.V.N.S.H., Sai Chandra R., Indian sign language recognition: A comparison between ANN and FIS, Journal of Theoretical and Applied Information Technology, Vol. 89, Issue. 2, 502 511, 2016.

24. Vijaya Lakshmi, V. S. Ghali, Muzammil Parvez M, G. V. P. Chandra Sekhar Yadav, V. Gopi Tilak, Fuzzy C-Means Clustering Based Anomalies Detection in Quadratic Frequency Modulated Thermal Wave Imaging, International Journal of Recent Technology and Engineering (IJRTE), Vol. 8 Issue. 3, 2019. https://doi.org/10.35940/ijrte.C5390.098319

25. G. V. Subbarao and R. Mulaveesala, Quadratic frequency modulated thermal wave imaging for Nondestructive testing, Progress in Electromagnetics Research M, Vol. 26, 11-22, 2012.

https://doi.org/10.2528/PIERM12062101

26. Hazel Mae A. Adriano, Dustin A. Reyes, Kristelee Jem B. Tan, Daniel D. Zagada, Reggie C. Gustilo, Defensive Turret Defensive Turret with Fully Automated Motion Detection Using Infrared Technology, International Journal of Emerging Trends in Engineering Research, Volume 7, No. 9 September 2019. https://doi.org/10.30534/ijeter/2019/05792019
27. Eko Julianto, Waluyo Adi Siswant, Marwan Effendy, Characteristics of Temperature changes and Stress of Float Glass under Heat Radiation, International Journal of Emerging Trends in Engineering Research, Volume 7, No. 9 September 2019. https://doi.org/10.30534/ijeter/2019/03792019 